\title{
Evidence based management of childhood urinary tract infections
}

\author{
Vindya Gunasekera ${ }^{1}$ \\ Sri Lanka Journal of Child Health, 2010; 39: 104-109
}

(Key words: Urinary tract infection, children, management)

\section{Introduction}

Urinary tract infection (UTI) is one of the common childhood bacterial infections. It affects $3.6 \%$ of boys and $11.3 \%$ of girls. Majority of them recover completely without long term sequelae. In the past it was thought that a significant proportion of children with UTI have structural abnormalities of the urinary tract which predispose them to recurrent infections. In addition, pyelonephritis and reflux nephropathy were believed to be major causes of chronic renal failure in children with high risk of hypertension and pregnancy associated complications in adulthood. Therefore, every young child after his or her first attack of UTI was exposed to a battery of predetermined radiological investigations to identify abnormalities. In the event of an anomalous imaging test this was followed by long term pharmacological therapy or corrective surgery to prevent further infection. Majority of these children with even minor structural abnormalities underwent the same imaging test more than once routinely, without much consideration to the invasive nature of these tests. Unfortunately, a significant proportion of them carried doubtful diagnoses of the first attack as well as recurrent attacks of UTI. Most of these older recommendations, in general, were based on expert opinion rather than solid scientific evidence derived from past studies which comprised highly selected populations with severe complicated disease $^{1}$.

However, during the past 2 decades, the management strategies of UTI have been greatly challenged. Doubts were raised regarding the prevalence of structural anomalies, their association with recurrent infections, as well as the significance of preventing infections to halt the long term sequelae. The recent guidelines from the National Institute for Health and Clinical Excellence (NICE) in UK published in 2007 recommend a more selective approach based on evidence $^{2}$. While some of the subsequent research

${ }^{1}$ Consultant Paediatric Nephrologist, Lady Ridgeway Hospital for Children, Colombo studies justified the NICE guidelines there are other studies which revealed contradictory results. The aim of this review is to evaluate the management strategies of childhood UTI based on appropriate descriptions and studies in the literature.

\section{Diagnosis of UTI}

UTI poses a diagnostic dilemma all around the world. This is extremely difficult in the preverbal child whose symptoms are very non specific thus necessitating a high index of suspicion. Fever is one of the commonest symptoms and may be the only symptom in the majority. Pooled prevalence of UTI among febrile children between the ages of 2 to 24 months is around $5 \%^{3}$. Table 1 summarizes the symptoms and signs suggestive of UTI in children ${ }^{2}$.

Criteria to screen for UTI according to the recommendations of American Academy of Pediatrics $^{3}$ and NICE guidelines ${ }^{2}$ are:

1. Children with symptoms and signs of UTI.

2. Unexplained fever of $\geq 38^{\circ} \mathrm{C}$ after 24 hours at the latest.

3. Those with an alternative site of infection who remain unwell after 24 hours at the latest.

(All the sites of infection such as ear and throat should be explored before labelling as unexplained fever).

It is also important to localize the infection to decide on acute as well as long term management plans. Table 2 highlights the features that help to differentiate upper tract (pyelonephritis) and lower tract (cystitis) infections. DMSA is the gold standard for localization of infection to renal parenchyma; however it is not recommended routinely for this purpose alone. 
Table 1

Symptoms and signs suggestive of UTI in children ${ }^{2}$

\begin{tabular}{|c|c|c|c|c|}
\hline \multirow{2}{*}{\multicolumn{2}{|c|}{ Age group }} & \multicolumn{3}{|c|}{ Symptoms and signs } \\
\hline & & Most common & & Least common \\
\hline \multicolumn{2}{|c|}{ Infants below 3 months } & $\begin{array}{l}\text { Fever } \\
\text { Vomiting } \\
\text { Lethargy } \\
\text { Irritability }\end{array}$ & $\begin{array}{l}\text { Poor feeding } \\
\text { Failure to thrive }\end{array}$ & $\begin{array}{l}\text { Abdominal pain } \\
\text { Jaundice } \\
\text { Haematuria } \\
\text { Odorous urine }\end{array}$ \\
\hline \multirow{2}{*}{$\begin{array}{l}\text { Infants and } \\
\text { children above } \\
3 \text { months }\end{array}$} & Preverbal & Fever & $\begin{array}{l}\text { Abdominal pain } \\
\text { Loin tenderness } \\
\text { Vomiting } \\
\text { Poor feeding }\end{array}$ & $\begin{array}{l}\text { Lethargy } \\
\text { Irritability } \\
\text { Haematuria } \\
\text { Odorous urine } \\
\text { Failure to thrive }\end{array}$ \\
\hline & Verbal & $\begin{array}{l}\text { Frequency } \\
\text { Dysuria }\end{array}$ & $\begin{array}{l}\text { Dysfunctional voiding } \\
\text { Incontinence } \\
\text { Abdominal pain } \\
\text { Loin tenderness }\end{array}$ & $\begin{array}{l}\text { Fever } \\
\text { Malaise } \\
\text { Vomiting } \\
\text { Haematuria } \\
\text { Odorous urine } \\
\text { Turbid urine }\end{array}$ \\
\hline
\end{tabular}

Table 2

The features that help to differentiate upper tract (pyelonephritis) and lower tract (cystitis) infections

\begin{tabular}{|l|l|}
\hline \multicolumn{1}{|c|}{ Pyelonephritis } & \multicolumn{1}{|c|}{ Cystitis } \\
\hline Young age $<2$ yrs & Older age $>2$ yrs \\
\hline Fever & Voiding problems \\
\hline Loin pain & Supra pubic pain \\
\hline High CRP & \\
\hline $\begin{array}{l}\text { Renal involvement i.e. high serum creatinine, } \\
\text { abnormal doppler USS, or DMSA }\end{array}$ & \\
\hline
\end{tabular}

\section{Collection of urine for analysis}

A clean catch midstream urine sample is the recommended method of collection. However when this is not practical a catheter or a supra pubic collection should be used ${ }^{2,3}$.

\section{Methods of screening and confirmation of UTI}

1. Urinalysis by dipstix for leucocyte esterase (LE), and nitrite.

2. Urinalysis by microscopy for leucocytes and gram stain for bacteria.

Combined urinalysis by dipstix and microscopy are reported to have a sensitivity and specificity of $80 \%$ each $^{3}$. Therefore urinalysis of a properly collected urine sample (clean catch, catheter or supra-pubic collection) is recommended as a good screening test. However applicability of urinalysis is still limited in Sri Lanka by availability and affordability.
1. Microscopy $(>10 \mathrm{WBC} / \mathrm{hpf}+$ moderate bacteria) has the highest positive likelihood ratio to rule in disease below the age 2 years.

2. Dipstick has highest positive likelihood ratio to rule in disease after the age of 2 years.

3. Microscopy ( $>5 \mathrm{WBC} / \mathrm{hpf}+$ few bacteria) has the lowest negative likelihood ratio to rule out UTI in all age groups.

Diagnosis of UTI is confirmed by demonstrating significant colony counts by culturing a properly collected urine sample. It is recommended to collect urine for culture before starting antibiotics for a child with suspected UTI or any serious illness. Criteria for significant bacteriuria, depends on method of collection (Table 3). 
Table 3

Criteria for significant bacteriuria as per method of collection ${ }^{3}$

\begin{tabular}{|c|l|l|}
\hline Method of collection & \multicolumn{1}{|c|}{ Colony count (pure growth) } & \multicolumn{1}{|c|}{ Probability of infection } \\
\hline Clean catch & & \\
Boy & $>10^{4}$ & Infection likely \\
& 3 samples $\geq 10^{5}$ & $95 \%$ \\
& 2 samples $\geq 10^{5}$ & $90 \%$ \\
& 1 sample $\geq 10^{5}$ & $80 \%$ \\
& $5 \times 10^{4-5}$ & Suspicious, repeat \\
\hline Suprapubic aspiration & Gram negative bacilli: any number & $99 \%$ \\
& Gram positive cocci: $>10^{3}$ & \\
\hline Transurethral catheterisation & $>10^{5}$ & $95 \%$ \\
& $10^{4-5}$ & Infection likely \\
& $10^{3-4}$ & Suspicious, repeat \\
& $<10^{3}$ & Infection unlikely \\
& $($ can increase sensitivity by taking & \\
& second 2-4 ml of urine) & \\
\hline
\end{tabular}

\section{Atypical UTI ${ }^{2}$ includes children:}

- who are seriously ill

- with poor urine flow

- with abdominal or bladder mass

- with raised creatinine

- with septicaemia

- who fail to respond to therapy within 48 hours

- *who are infected with non E coli organisms

*UTI is generally caused by gram negative coliforms with E coli accounting for more than $80 \%$ of cases. Non E coli pathogens are associated with complicated UTI in some studies.

\section{Definition of recurrent $\mathbf{U T I}^{2}$}

1. Two or more episodes of acute pyelonephritis/ upper urinary tract infection or

2. One episode of acute pyelonephritis plus one or more episodes of cystitis or

3. Three or more episodes of cystitis

\section{Treatment of an acute episode}

The aim of treatment with antibiotics is 1) to eradicate infection, 2) to relieve symptoms and 3) to minimize parenchymal defects. Therefore, after an appropriate collection of a urine specimen for culture, antibiotics should be started as early as possible especially for infants and children below 2 years suspected to have febrile UTI (preferably within 24 hours). However, the treatment should not be delayed if urine is unobtainable. Even though the risk of getting renal parenchymal damage is minimal following acute cystitis, prompt treatment is indicated 1) to relieve symptoms and 2) to prevent possible progression to pyelonephritis. The choice of antibiotic, route of administration and the duration of treatment will depend on a combination of factors.

\section{NICE recommendations for treatment of UTI in children $^{2}$}

1. For infants below 3 months of age with suspected UTI: parenteral antibiotics are recommended for a duration of 7-10 days.

2. For infants and children above 3 months of age with acute pyelonephritis:

- Treat with oral antibiotics for 7-10 days.

- If oral antibiotics cannot be used parenteral antibiotics are recommended for 2-4 days, followed by oral antibiotics for a total duration of 10 days.

- If aminoglycosides are used once daily dosing is recommended.

3. For infants and children above 3 months of age with acute cystitis: oral antibiotics for 3 days are recommended.

It is evident that these recommendations were based on the best available literature. A Cochrane review $^{4}$ included 23 randomised and quasirandomised controlled trials which involved 3407 children with acute pyelonephritis who received either oral antibiotic for 10-14 days or initial IV antibiotics for 3 days followed by oral antibiotics for 10 days. There were no significant differences in 1) duration of fever, 2) persistent UTI at 72 hours, 3) recurrence of UTI within 6 months or 4) persistent renal damage at $6-12$ months. However, there had been exclusion criteria in most of these studies e.g. renal impairment, severe urinary tract anomaly, sensitivity to study medications, recent 
antibiotic use, previous UTI, signs of shock at presentation, and immune compromised patients.

Since NICE recommendations were meant for children with simple UTI, parenteral antibiotics should still be indicated for those outside the scope of these guidelines with more complicated uropathologies, those with atypical UTI and also for whom there are significant practical difficulties in administering oral medication like young infants.

Limited data is available regarding the type of antibiotic to be used and all the antibiotics are generally accepted as suitable. Therefore, when prescribing empirical treatment for UTI, it is advised to follow the local sensitivity patterns and also to prescribe drugs which are least susceptible to develop resistance.

Antibiotics suggested in the NICE guidelines ${ }^{2}$ are:

1. Parenteral antibiotics: co-amoxyclav, 3rd generation cephalosporins, aminoglycosides.

2. Oral antibiotics to treat pyelonephritis: coamoxyclav, cephalosporin.

3. Oral antibiotics to treat cystitis: Trimethoprim, nitrofurantoin, cephalosporin, amoxicillin.

As empirical treatment of simple febrile UTI, some centres prefer to use aminoglycosides instead of cephalosporins, to minimize emergence of resistant strains. Single daily dosing of aminoglycosides have been shown to be beneficial to minimize the cost, simplify administration, and also to provide similar or even improved efficacy and safety, when compared to multiple daily dosing, in infections other than UTI as well. In neonates it is recommended to combine with ampicillin. However usage of this drug should be limited to patients with simple febrile UTI without renal parenchymal or uropathological abnormality. Monitoring of renal function is highly recommended during treatment.

\section{Imaging in UTI}

Aim of imaging in children with UTI is to identify underlying structural anomaly/anomalies that predispose them to recurrent infections and kidney injury because it was thought that UTI may be the first indicator of an underlying congenital abnormality of the genitourinary tract. In addition, pyelonephritis and reflux nephropathy were believed to be major causes of chronic renal failure in children with high risk of hypertension and pregnancy associated complications in adulthood.
These theories are not supported by other studies which showed that clinically adverse outcomes probably occur only in a few cases and severe bilateral renal scarring and pyelonephritic scarring detected in DMSA scan are most likely secondary to congenital dysplasia ${ }^{5}$. Claesson et al in 1981 found that the parenchymal defects in kidneys get smaller with time and the compensatory growth of healthy kidney tissue result in mean renal area approaching $98 \%$ of normal after 15 years ${ }^{6}$. Moorthy et al demonstrated lower rates of vesicoureteric reflux (VUR) among children with UTI than previously described and showed that VUR is not a useful way to identify infants at risk of scarring ${ }^{7}$.

Previous guidelines were formulated using age as the main factor to determine the follow up investigations leading many children to undergo invasive imaging studies and also posing heavy burden on resources ${ }^{8}$. NICE guidelines $(2007)^{2}$ put forward a more selective approach targeting only infants and children belonging to an at risk group, thus causing a significant reduction in radiological investigations.

\section{NICE $(2007)^{2}$ recommendations:}

\section{Indications for USS}

1. $<6$ months of age: all children

2. 6 months of age or $>$ : only atypical or recurrent UTI

\section{Indications for DMSA (4-6 months after UTI)}

1. $<6$ months of age: atypical or recurrent UTI

2. 6 months of age or $>$ : only recurrent UTI

\section{Indications for $M C U G$}

1. <6months of age: abnormal USS

2. 6 months to 3 yrs of age: dilatation in USS, poor urine flow, non $\mathrm{E}$ coli infection or those who have family history of VUR

3. $>3$ yrs; not indicated

The restrictions were lightened for young infants since studies have shown that young infants presenting with UTI have a higher likelihood of having obstruction and major urological problems. Further, the young growing kidney is thought to be vulnerable to insults and it is accepted that infants run the greatest risk of developing scarring after UTI, this risk decreasing significantly after the age of 5 years ${ }^{9}$. It is still accepted in all contexts that children with recurrent UTI need detailed evaluation since the risk of renal scarring increases as the number of UTI recurrences increase ${ }^{9}$. 
However, Niko et al $(2009)^{8}$ showed that a significant proportion of patients with reflux and scarred kidneys are missed if NICE guidelines are followed. In NICE guidelines it was decided not to investigate most of the children with simple UTI, since there was no high level evidence on diagnostic value and clinical effectiveness of detecting minor structural abnormalities as well as the association of VUR and scarring ${ }^{5,7}$. However, there is not enough evidence to disregard significant pyelonephritic scarring as the cause of conventionally believed long term sequelae either. In fact, it may have been invariably assumed that in UK major structural abnormalities were detected during routine antenatal USS.

\section{Local scenario}

1. No routine antenatal USS is available.

2. Lack of statistics of urological problems.

3. Incidence of late diagnosis of surgically correctable urological problems is not uncommon.

4. All children with first attack of UTI may not be true first timers.

Therefore, I think that we still have to adhere to the old practice of offering USS to all the children who present with UTI. In addition to the NICE recommendations, MCUG is indicated irrespective of the age, if there are features of bladder outflow obstruction or neurogenic bladder. It is also indicated for those with recurrent /atypical UTI on an individualized basis and may be for those with significant voiding problems, since in Sri Lanka there are minimal facilities to do urodynamic studies in children.

Recently, a DMSA has been advocated in the first place and MCUG being indicated for those with abnormal DMSA. According to my understanding of the current literature, a DMSA is preferably indicated for infants with febrile UTI and for those with atypical or recurrent UTI above infancy. However, this may be limited by its affordability and availability.

\section{Antibiotic prophylaxis}

UTI is the most frequent reason for prophylactic antibiotics in infants and children. Studies have shown that children are the major consumers of antibiotics with usage rates three times higher than older patients ${ }^{10}$ thereby increasing their risk of developing resistant organisms. It is stated that for patients infected with multidrug resistant organisms we have reached a point as frightening as the pre- antibiotic era ${ }^{10}$. Therefore, this major public health problem should be kept in mind when selecting antibiotics to treat acute attacks of UTI and also when deciding to start long term prophylaxis.

\section{NICE recommendations to start prophylactic antibiotics:}

1. Should not be recommended after first attack of UTI.

2. May be considered for recurrent infections.

These indications were based on findings of previous small heterogeneous studies which did not demonstrate any difference in new scar formation, deterioration of renal parenchymal defects or any high level evidence of reduction of symptomatic UTI following antibiotic prophylaxis ${ }^{2}$. Three multicentre prospective, randomized controlled trials in 2008 also failed to demonstrate a clear benefit of prophylaxis following a first febrile UTI in the absence of or with low grade VUR. These were again small studies and children with major structural problems were excluded and therefore there were no definite conclusions for higher grade reflux. However, 3 randomized controlled trials are yet to be published 2 of which are double blind multicentre and placebo controlled studies which might address the unanswered issues. Pending the results of these studies, drastic changes to the recommendations of prophylaxis should also be guarded. It is wise to make individualized decisions for the high risk patients, e.g. young infants, recurrent infections, following severe infection, pre-existing significant pyelonephritic scarring and dilating VUR. However, when selecting antibiotics, options should be limited to nitrofurantoin, nalidixic acid and cotrimoxazole. Cephalosporins should be avoided except in infants below 2 months of age.

\section{Surgical correction for VUR}

Past studies had demonstrated that children with persistent VUR are at a higher risk of developing hypertension at adulthood ${ }^{11}$ and also that the female child with persistent VUR is at a higher risk of getting new scars in adulthood ${ }^{12}$. Systematic review of 11 studies $^{13}$ have failed to demonstrate any significant difference in terms of recurrent UTI, end stage renal failure at 5 years, hypertension at 5 and 10 years or new or progressive parenchymal abnormalities in children who received either prophylactic antibiotic and surgical correction or antibiotic alone. Even though risk of clinical pyelonephritis was $50 \%$ less in the surgically managed group there was no concomitant reduction of progressive renal damage in 10 years. However, there had been no 
comparison between groups with similar grade reflux and grade $\mathrm{V}$ reflux was excluded. Furthermore, the association of surgical complications such as ureteric stenosis and also the experience of the surgeon were not considered.

\section{Conclusion}

Most of the long term management issues still remain un-answered. More emphasis should be focused on early identification and accurate diagnosis of UTI followed by proper clinical evaluation to exclude major urological abnormalities. Most of the children with simple febrile UTI can be treated with oral antibiotics alone but parenteral antibiotics may be indicated at least initially for patients at risk of getting renal parenchymal injury. Children beyond infancy with the first attack of simple febrile UTI may not need invasive and costly testing other than USS or intensive follow up including prophylaxis. Furthermore, paediatricians need to make intelligent clinical decisions to avoid invasive investigations in children when the diagnosis of UTI is doubtful. However, proper guidelines are yet to be published.

\section{References}

1. Hodson CJ, Edwards D. Chronic pyelonephritis and vesicoureteric reflux. Clin Radiol 1960; 11:219-31.

2. National Institute for Health and Clinical Excellence. Urinary tract infection in children: Diagnosis treatment and long term management. Available from: 2007 http//www.nice.org.uk/nicemedia/pdf/

3. American Academy of Pediatrics: Practice parameter: The diagnosis, treatment and evaluation of the initial urinary tract infection in febrile infants and young children Pediatrics 1999; 103: 843-52.

4. Hodson EM, Willis NS, Craig JC. Antibiotics for acute pyelonephritis in children. Cochrane Database Syst Rev. 2007 Oct 17; (4): CD003772.

5. Risdon RA, Yeung CK, Ransley PG, Reflux nephropathy in children submitted to unilateral nephrectomy: a clinic-pathological study. Clin Nephrol1993; 40(6):308-14.
6. Claësson I, Jacobsson B, Jodal U, Winberg J. Compensatory kidney growth in children with urinary tract infection and unilateral renal scarring: an epidemiologic study. Kidney Int 1981; 20(6):759-64.

7. Moorthy I, Easty M, McHugh K, Ridout D, Biassoni L, Gordon I. The presence of vesicoureteric reflux does not identify a population at risk for renal scarring following a first urinary tract infection. Arch Dis Child 2005; 90(7):733-6.

8. Tse NK, Yuen SL, Chiu MC, Lai WM, Tong PC. Imaging studies for first urinary tract infection in infants less than 6 months old: can they be more selective? Pediatr Nephrol 2009; 24(9):1699-703.

9. Orellana P, Baquedano P, Rangarajan V, Zhao JH, Eng ND, Fettich J, et al Relationship between acute pyelonephritis, renal scarring, and vesicoureteral reflux. Results of a coordinated research project. Pediatr Nephrol 2004; 19(10):1122-6.

10. Montini G, Hewitt I. Urinary tract infections: to prophylaxis or not to prophylaxis? Pediatr Nephrol 2009 Sep; 24(9):1605-9.

11. Smellie JM, Prescod NP, Shaw PJ, Risdon RA, Bryant TN. Childhood reflux and urinary infection: a follow-up of 10-41 years in 226 adults. Pediatr Nephrol 1998; 12(9):727-36.

12. Martinell J, Hansson S, Claesson I, Jacobsson B, Lidin-Janson G, Jodal U. Detection of urographic scars in girls with pyelonephritis followed for 13-38 years. Pediatr Nephrol 2000; 14(10-11):1006-10.

13. Hodson EM, Wheeler DM, Vimalchandra D, Smith GH, Craig JC. Interventions for primary vesicoureteric reflux. Cochrane Database Syst Rev. 2007 July 18; (3):CD001532. 